\title{
Active Mode Switching in Plasmonic Microlasers by Spatial Control of Optical Gain
}

\author{
Robert C. Keitel,,$^{\dagger}$ Marianne Aellen,,$^{\dagger}$ Boris le Feber, ${ }^{\dagger}$ Aurelio A. Rossinelli, ${ }^{\dagger}$ \\ Stefan A. Meyer, ${ }^{\dagger}$ Jian Cui, ${ }^{\dagger}, \dot{+}$ and David J. Norris ${ }^{*}, \dot{\dagger}$
}

${ }^{\dagger}$ Optical Materials Engineering Laboratory, Department of Mechanical and Process Engineering, ETH Zurich, 8092 Zurich, Switzerland

tHelmholtz Pioneer Campus, Helmholtz Zentrum München, Ingolstädter Landstraße 1, 85764 Neuherberg, Germany

\section{Contents}

Section

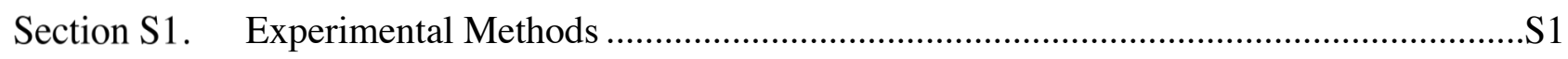

Section S2. Additional Characterization of Plasmonic Lasing ..............................................S4

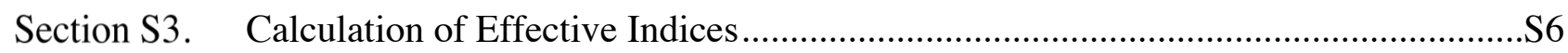

Section S4. Impact of the Threshold Parameter in Pump-Profile Generation ............................S6

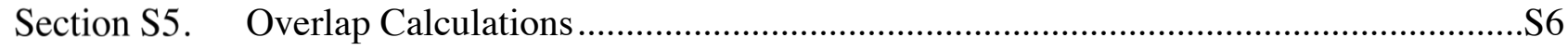

Section S6. Impact of Pump-Pattern Misplacement ............................................................

Section S7. Lasing Threshold with Patterned Pumping .......................................................S8

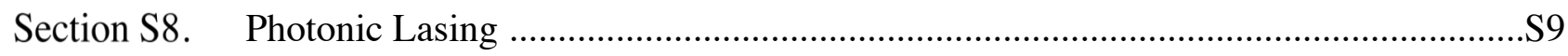

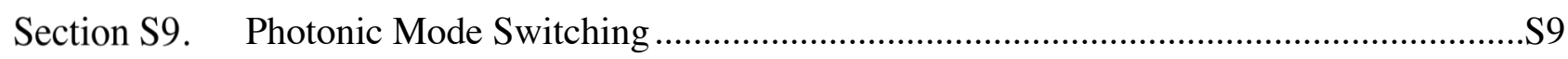

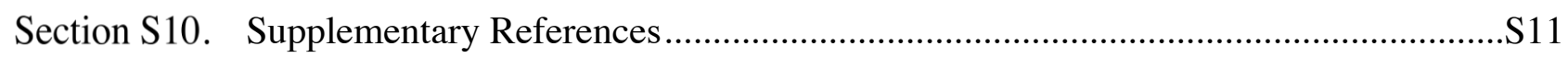

\section{Section S1. Experimental Methods}

Materials. Acetone (Technic France, Micropur VLSI Grade), isopropanol (IPA, Technic France, Micropur VLSI Grade), CSAR resist (AR-P 6200.04, Allresist GmbH), developer solution (AR 600-546, Allresist $\mathrm{GmbH}$ ), and buffered hydrofluoric acid (hydrogen fluoride/ammonium fluoride 1:7, Technic France, Micropur VLSI Grade) used for template fabrication were provided by the Binnig and Rohrer Nanotechnology Center (BRNC) at IBM Zurich. Nitric acid $\left(65 \% \mathrm{HNO}_{3}\right)$ was purchased from Fisher Chemicals. Deionized water was obtained from a MilliQ Advantage A10 water purification system. Silver (Ag, 99.99\%) pellets and tungsten 
boats were purchased from Kurt Lesker and Umicore, respectively. Ultraviolet-curable epoxy (OG145-95) was purchased from Epoxy Technology.

Plasmonic cavity fabrication. Si templates (1-mm-thick, 2-inch-diameter, $<100>$ ) for template stripping of the plasmonic cavities were fabricated similar to a procedure described in a previous paper. ${ }^{\mathrm{S} 1}$ Wafers were cleaned by ultrasonication for $2 \mathrm{~min}$ in acetone and IPA, and dried with nitrogen gas. The wafers were prebaked for $10 \mathrm{~min}$ at $180^{\circ} \mathrm{C}$ and allowed to cool to room temperature. A layer of CSAR resist was applied via spin coating at $2000 \mathrm{rpm}$ for $60 \mathrm{~s}$, followed by a postbake step $\left(150^{\circ} \mathrm{C}\right.$ for $\left.5 \mathrm{~min}\right)$. The desired pattern was exposed using an electron beam lithography system (Vistec, EBPG5200). After exposure, the resist was developed for $1 \mathrm{~min}$ in the developer solution, and the pattern was transferred into the Si by reactive-ion etching (Oxford Instruments, Plasmalab System 100). The remaining resist was removed by ultrasonication in acetone and IPA, followed by 2 min of $\mathrm{O}_{2}$-plasma cleaning at $600 \mathrm{~W}$, a dip in buffered hydrofluoric acid, and rinsing with DI water.

Immediately before use, templates were cleaned by submersion in nitric acid, followed by rinsing and sonication in deionized water and IPA. Cleaned templates were mounted on a homebuilt rotating sample holder in a thermal evaporator (Kurt J. Lesker, NANO 36). $1 \mu \mathrm{m}$ of Ag was deposited at a rate of $25 \AA / \mathrm{s}$ under an initial chamber pressure of $\sim 3 \times 10^{-7}$ Torr. Subsequently, microscope glass slides were bonded to the Ag surface using an ultraviolet-curable epoxy. Immediately after manual template stripping, 100 atomic-layer deposition cycles of alumina were deposited at $50{ }^{\circ} \mathrm{C}$ (Picosun, Sunale R-150). Following template stripping, the templates can be cleaned and reused multiple times.

Nanoplatelet synthesis. Colloidal core/shell/shell $\mathrm{CdSe} / \mathrm{Cd}_{\mathrm{x}} \mathrm{Zn}_{1-\mathrm{x}} \mathrm{S} / \mathrm{ZnS}$ nanoplatelets were synthesized following a previously published recipe. ${ }^{\mathrm{S} 2}$

Nanoplatelet printing. Colloidal nanoplatelets were printed into the cavities using a previously published procedure. ${ }^{\mathrm{S3}}$ To achieve plasmonic lasing, we performed multiple overprints of NPLs until a thickness of 45$50 \mathrm{~nm}$ was reached. The appropriate print parameters were determined from a parameter sweep followed by optical microscopy to estimate the film thickness. ${ }^{\mathrm{S} 3}$ Film thicknesses were verified by atomic force microscopy (Bruker, Dimension FastScan) after all optical measurements were completed.

Optical characterization and selective pumping. For plasmonic lasing measurements, samples were mounted in a closed-cycle helium cryostat (Montana Cryostation 2 with LWD option) on a piezo positioning system (Attocube, ANPz101, and two ANPx101). Optical characterization was performed using an ultrafast 


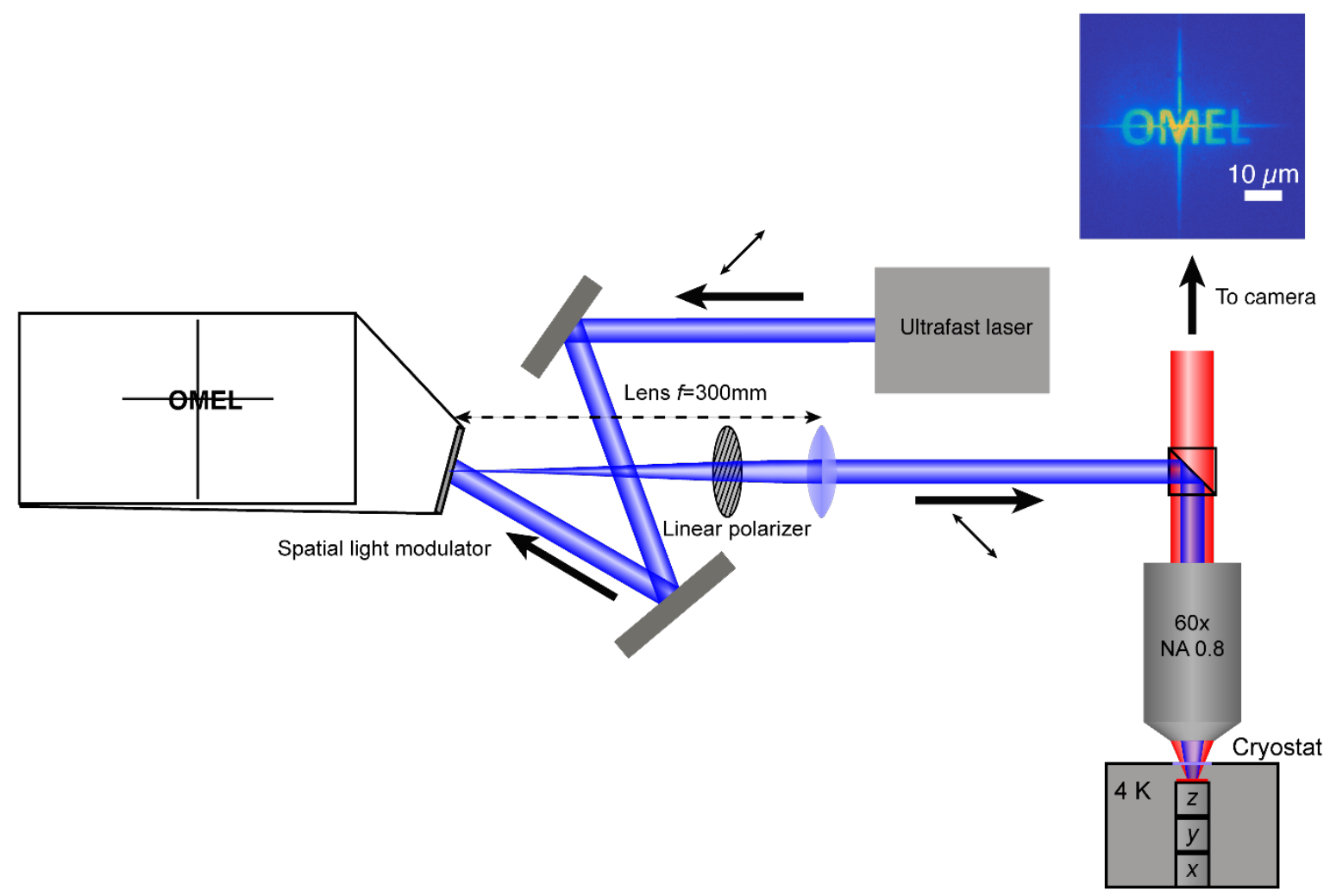

Figure S1. Schematic of the setup used for excitation patterning. Thick arrows indicate the direction of light propagation and double arrows the axis of linear polarization.

pulsed laser source ( $480 \mathrm{~nm}$; pulse duration $\sim 340 \mathrm{fs}$; repetition rate $100 \mathrm{~Hz}$ ) consisting of a collinear optical parametric amplifier (Spectra-Physics, Spirit-OPA) pumped by a $1040 \mathrm{~nm}$ pump laser (Spectra-Physics, Spirit-1040-8). The beam intensity was adjusted using a neutral density filter wheel (Thorlabs, NDC-50C-2MB). Following expansion and collimation, a fraction of the beam was directed onto a photodiode (Thorlabs, S120VC) connected to a power meter (Thorlabs, PM100D). The measured power was later correlated with the power measured by a second photodiode (Thorlabs S170C) connected to a second power meter placed on top of the microscope stage to calculate the fluence at the sample.

The remaining portion of the beam was shaped with the use of a reflective spatial light modulator (SLM, HOLOEYE PLUTO NIR-11). A sketch of the setup can be found in Figure S1. For this, the incident beam was polarized at $45^{\circ}$ with respect to the SLM horizontal axis using a linear polarizer (Thorlabs LPVISE100A). Placing a second linear polarizer at $-45^{\circ}$ behind the SLM allows for amplitude modulation. An imaging lens (Thorlabs AC254-300-A-ML) with the SLM in the focal plane, in combination with a 60x objective lens [Nikon, CFI S Plan Fluor ELWD, numerical aperture (NA) 0.7], was used to project the SLM pattern onto the sample. A dichroic beam splitter (488-nm long pass, AHF analysentechnik) was used to separate excitation from emission. A longpass filter (Thorlabs, FELH-0600) further suppressed pump-laser leakage. With this setup, arbitrary patterns with sub- $\mu \mathrm{m}$ resolution and placement accuracy below $100 \mathrm{~nm}$ could be generated. 
Weak excitation fluences for imaging were obtained by attenuating the pump power by two orders of magnitude using neutral density filters (Thorlabs, ND20A) with respect to the power used for lasing measurements to ensure an emission intensity proportional to the excitation and increasing the repetition rate of the laser to $10 \mathrm{kHz}$.

Emission from the sample was collected through the same objective, filtered by the dichroic and the longpass filter, and directed with relay lenses (focal length $200 \mathrm{~mm}$ ) into an imaging spectrometer (Andor, Shamrock 303i). Here, the emission was spatially filtered with a slit in the image plane closed along the dashed line in Figure 1b, dispersed by a 300 lines/mm grating, and imaged with an electron-multiplying chargecoupled device (EMCCD) camera (Andor, iXon 888 Ultra). Real-space images were obtained using the zerothorder diffraction of the same grating. An aperture in the intermediate image plane, in combination with a Wollaston prism in front of the last lens, splits the image into two images with horizontal and vertical polarization, respectively. Simultaneously measuring both polarization components allows us to quickly, and with high fidelity, identify whether a lasing mode is plasmonic (TM) or photonic (TE).

The relationship between dimensions on the SLM and dimensions on the sample was calibrated by generating a bright spot on a dark background on the SLM, which was then projected onto a film of NPLs. This image was then moved, using the SLM, along the horizontal and vertical axes. By determining the locations of these bright spots on the camera image relative to their corresponding locations on the SLM, we obtained a calibration from SLM pixels to pixels on the camera. Finally, camera space was converted to sample space by taking into account the magnification of our microscope.

\section{Section S2. Additional Characterization of Plasmonic Lasing}

The lasing threshold can be better observed when analyzing the peak intensity, rather than the integrated area. We extracted the height of the peaks corresponding to the cavity modes and to the background. We show how they evolve with pump fluence in Figure S2a. While the background grows sub-linearly over all powers investigated, both cavity modes show a super-linear growth starting around $14 \mu \mathrm{J} \mathrm{cm}^{-2}$. From the same dataset we also extracted the fluence-dependent linewidth of the lasing peaks (Figure S2b). While the peaks are barely visible below threshold, and the width cannot be estimated with certainty, we observe a slight reduction in linewidth above threshold. However, it is difficult to estimate the linewidth as long as the respective lasing peak is significantly smaller than the background. Well above threshold, the lasing lines start to broaden again. 
a

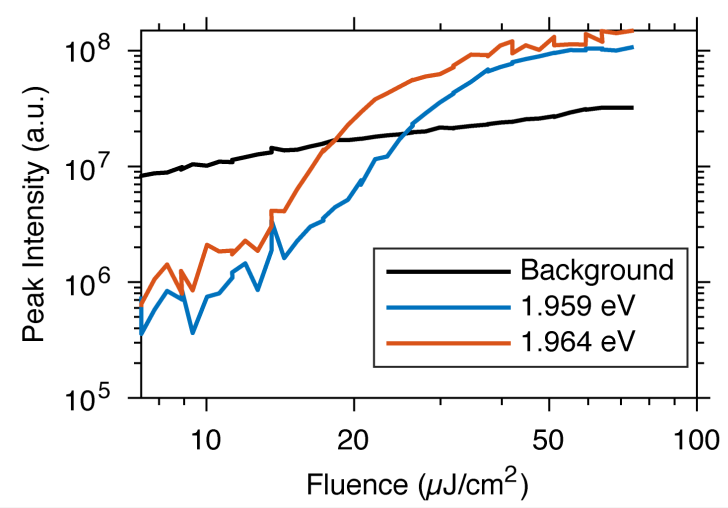

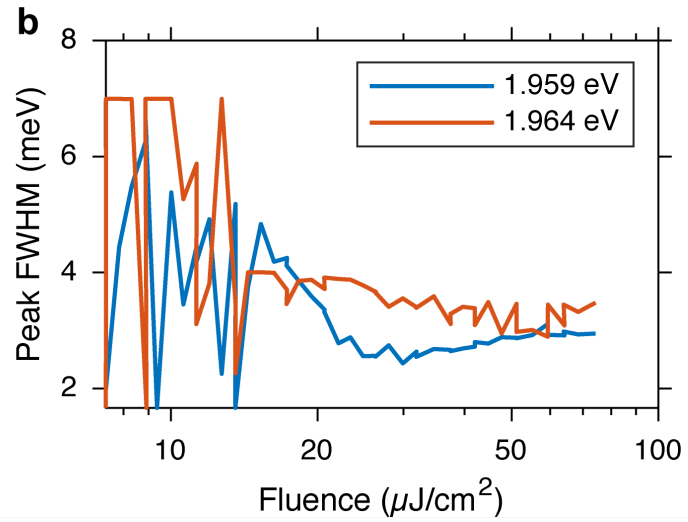

Figure S2. Extended characterization of the plasmonic laser. (a) Pump-fluence dependence of the peak intensities of the spontaneous emission and two dominant lasing peaks. (b) Pump-fluence dependence of the width of the two lasing peaks.
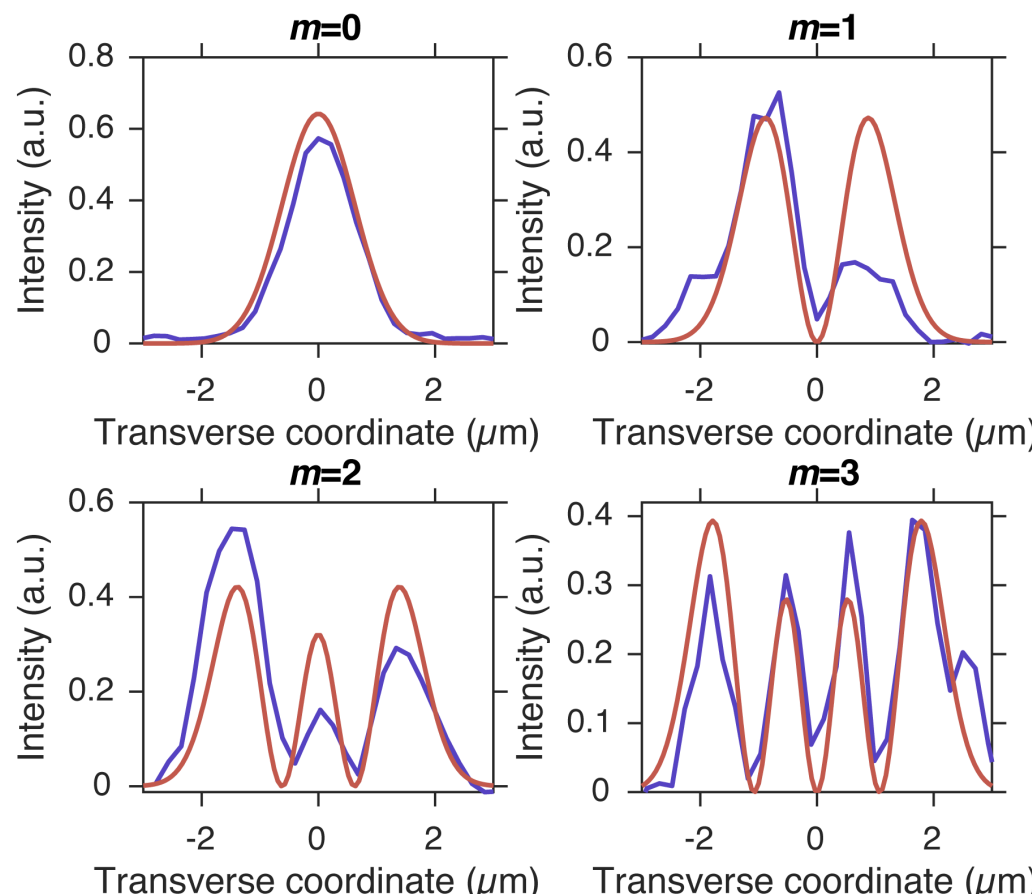

- Experiment

Transverse coordinate $(\mu \mathrm{m}) \quad$ Transverse coordinate $(\mu \mathrm{m})$

Figure S3. Beam characteristics of plasmonic lasing. Experimentally obtained transverse intensity profiles of the signal scattered at the reflector edge are compared with theoretical predictions for the first four transverse modes. The good agreement shows that the lasing modes form well-defined Hermite-Gauss beams.

Another criterion to demonstrate lasing is that the output forms a well-defined beam. ${ }^{\mathrm{S}}$ The signal we measure in our devices is due to scattering of the lasing mode at the reflector edge. In Figure S3, we compare the transverse intensity distribution of this scattered signal for different transverse modes with predictions based on eq 1 of the main text. The excellent agreement confirms that the lasing modes form well-defined beams. 


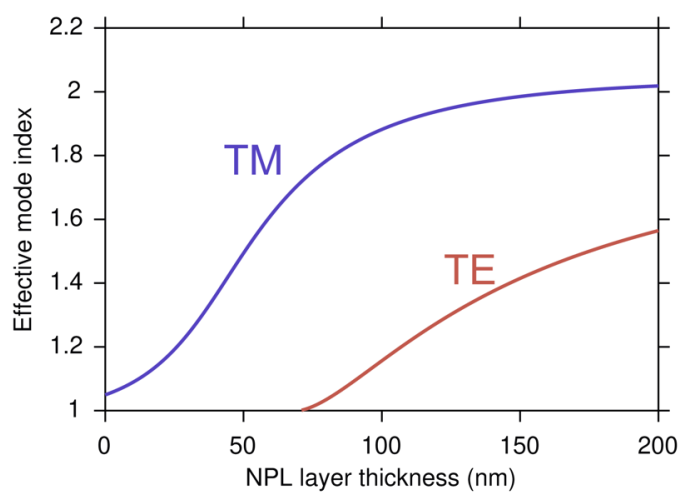

Figure S4. Calculated effective mode indices for TM and TE modes at $1.960 \mathrm{eV}$ for different thicknesses of the NPL gain layer.

\section{Section S3. Calculation of Effective Indices}

The effective indices for plasmonic (TM) and photonic (TE) modes were calculated using a multilayerwaveguide model. ${ }^{\mathrm{S} 3 \mathrm{~S} 5}$ The layer stack consisted of a $\mathrm{Ag}$ substrate, covered by a $10 \mathrm{~nm}$ protective alumina layer, and a film of nanoplatelets of varying thickness. Refractive indices of the respective layers were obtained from ellipsometry measurements. The resulting effective mode index at the experimentally observed resonance energy of $1.960 \mathrm{eV}$ is shown in Figure S4. For the plasmonic microlaser shown in the main text, we measured a NPL film thickness of $45-50 \mathrm{~nm}$, which corresponds to an effective mode index of 1.5. The photonic laser discussed in Sections S8 and S9 below had a NPL-film thickness of 90-100 nm and an estimated mode index of 1.1.

\section{Section S4. Impact of the Threshold Parameter in Pump-Profile Generation}

While the penalty coefficient mainly has an impact on the overlap with lower-order transverse modes, the threshold parameter mainly controls the lateral extent of the pump pattern. In Figure S5, we show the effect that this parameter has on the pump pattern and mode selectivity. If the threshold is too low, the overlap with unwanted modes becomes high enough to give multimodal lasing.

\section{Section S5. Overlap Calculations}

Linear overlap integrals were calculated by multiplying the binary optical modulation pattern with the calculated spatial intensity distribution of the respective plasmonic lasing modes (square modulus of eq 1 in the main text). Normalizing the spatial distributions of the modes to carry unity integrated intensity in the volume of the cavity guarantees that a uniform pump has unity overlap with each transverse mode, while no 
a

\section{threshold $=0.01$ $m=2$ modes dominate}

b

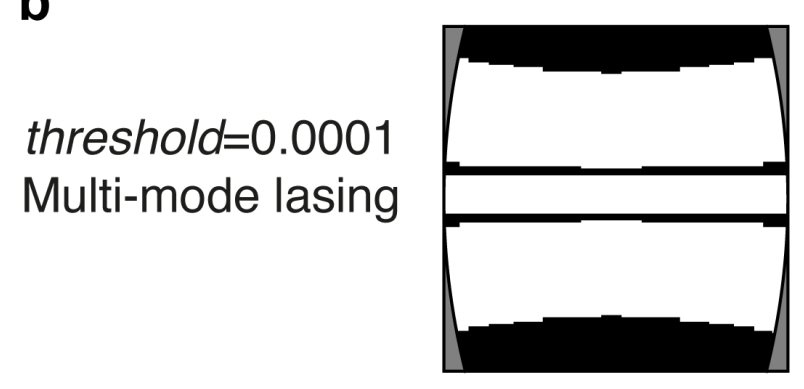

C

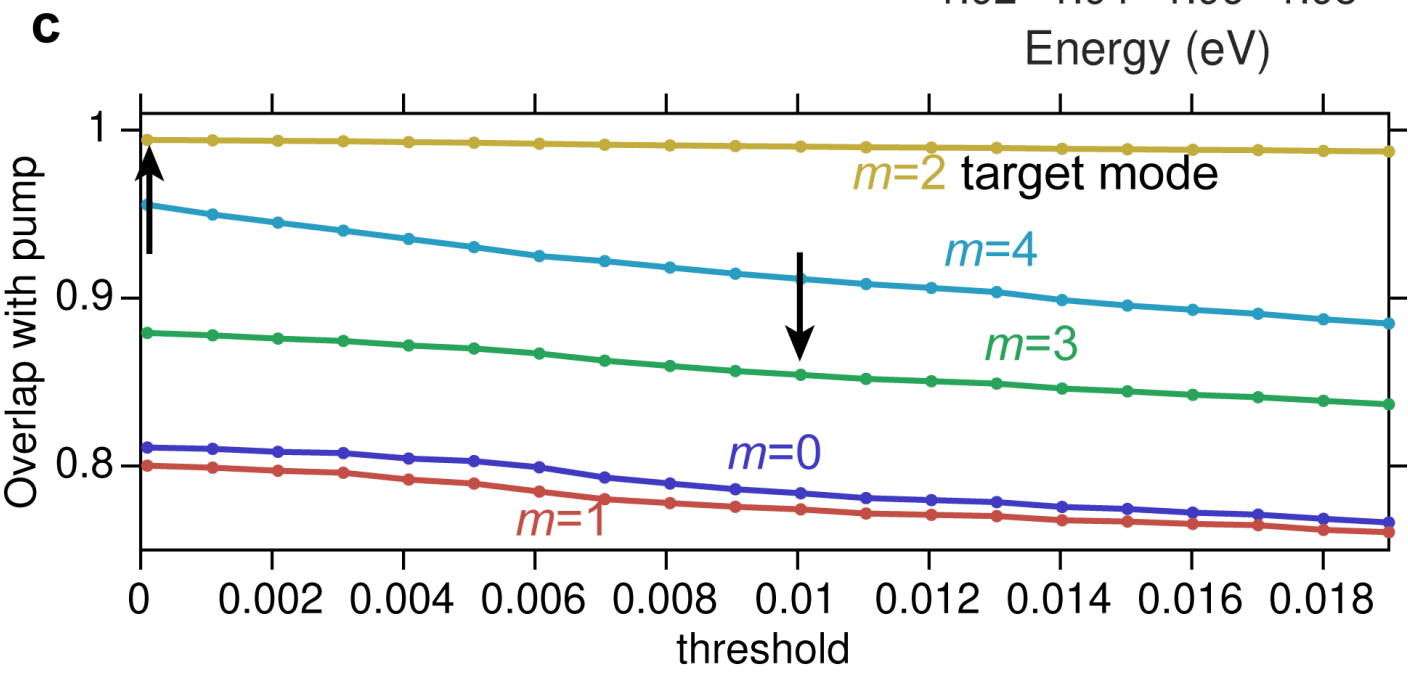

Figure S5. Impact of threshold parameter on mode switching. (a) Optical pumping of a plasmonic cavity with a pattern for lasing in the $m=2$ transverse mode with threshold $=0.01$ leads to a dominant narrow peak at $1.957 \mathrm{eV}$ on top of the spontaneous emission background. The small peak at $1.971 \mathrm{eV}$ is the next-higher longitudinal mode while the small peak at $1.962 \mathrm{eV}$ corresponds to a residual higher-order transverse mode that is not ideally suppressed. (b) If a lower threshold is chosen, the gaps in the pump profile extend further laterally, which makes higher-order modes more likely to lase. The additional lasing peaks correspond to lasing in the $m=3$ and $m=4$ modes. (c) These findings, as in Figure 4 in the main text, can again be explained by the linear overlap integral of the pump pattern targeting the $m=2$ mode with the different transverse modes.

pump has zero overlap. As shown in Figure 3 of the main text, our calculated linear overlap captures the observed phenomena in a good approximation of an ostensibly non-linear system. 

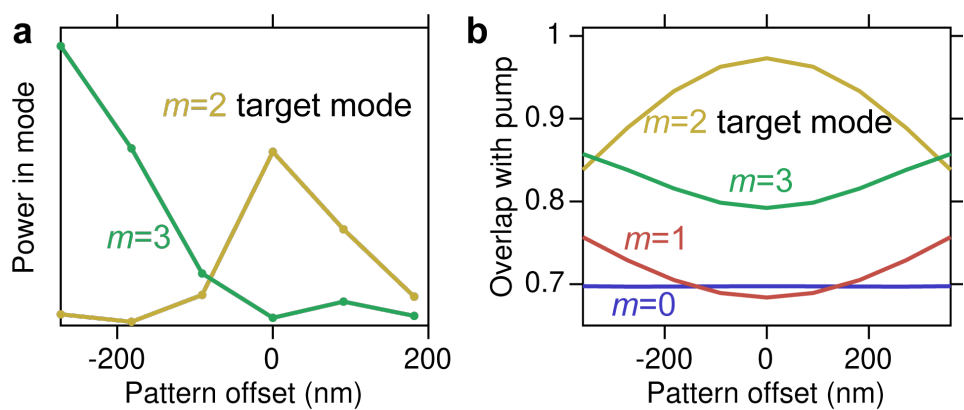

Figure S6. Impact of pump-pattern misplacement. (a) A lateral misalignment of as little as $100 \mathrm{~nm}$ drastically alters the laser output, leading to almost no lasing in the target mode. For displaced patterns, modes different from the targeted mode can become dominant. (b) The overlap integral of the pump pattern with the targeted mode monotonically drops when shifting the pump pattern. For an even-order targeted mode, odd-order modes in particular can experience a higher overlap, which explains the experimental findings in panel a.

\section{Section S6. Impact of Pump-Pattern Misplacement}

Accurate placement of the engineered pump profile on the cavity center axis is crucial for optimal performance. In Figure S6a, we show experimental results from a pattern that leads to single-transverse-mode lasing in the $m=2$ target mode when optimally placed. A lateral offset of as little as $100 \mathrm{~nm}$ by shifting the pattern by one pixel on the SLM significantly lowers the power in the targeted mode. It can even cause significant lasing in unwanted modes. The calculated overlap integral for the targeted mode rapidly drops with lateral displacement while the overlap of the pump with modes that have opposite parity shows a significant increase. This explains the observation of lasing in the $m=3$ mode for a displaced pattern (Figure S6b).

\section{Section S7. Lasing Threshold with Patterned Pumping}

The high overlap of the pump pattern with the targeted modes suggests that the lasing threshold should not be raised significantly. Note that in our approach of amplitude modulation, the peak fluence remains constant, independent of the pump pattern chosen. In Figure S7, we compare the peak intensity as a function of the pump fluence for a homogeneous pump and for profiles that target different transverse modes. While the threshold for homogeneous pumping for the investigated device occurs around $35 \mu \mathrm{J} \mathrm{cm}^{-2}$, we observe only a slight increase to approximately $40 \mu \mathrm{J} \mathrm{cm}^{-2}$ when targeting a specific mode. We highlight that the near-constant threshold arises because we report the pump fluence, a quantity normalized per area. While the area density of the pump energy remains nearly constant, the overall pump energy required to achieve lasing is reduced because we pump a smaller area. Ideally, we would only reduce the overall energy in the beam while maintaining the fluence in the cavity regions that are still pumped. Accordingly, if a tailored beam illuminates all areas where a given mode is localized, the threshold should not change at all. However, because our scheme 


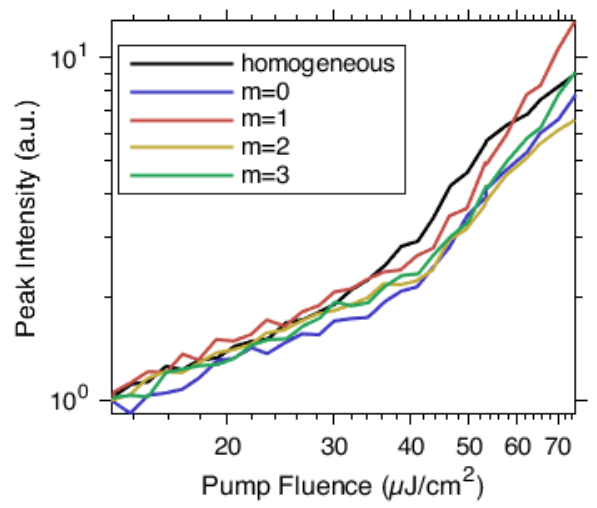

Figure S7. Impact of mode-targeting via pump profiles on the lasing threshold. The threshold is only slightly increased when targeting a specific mode compared to the case where the cavity is homogeneously pumped.

also requires us to avoid illuminating areas where the target mode is weak to suppress unwanted modes, we see a slight increase in the lasing threshold. This is in agreement with our experimental observations.

\section{Section S8. Photonic Lasing}

When using a NPL-film thickness above the cut-off thickness for photonic modes, we see a similar evolution of the output spectrum with pump fluence, transitioning from spontaneous emission to lasing peaks (Figure S8a). However, a pronounced super-linear growth of the output power in the TE-polarized channel at $\sim 20 \mu \mathrm{J} \mathrm{cm}^{-2}$ (Figure S8b) clearly indicates the photonic nature of the lasing modes. In agreement with previous observations, the "kink" at the threshold is much more visible due to the less efficient coupling of the NPL spontaneous emission to the cavity mode. ${ }^{\mathrm{S} 3}$

\section{Section S9. Photonic Mode Switching}

Figure S9 shows data similar to that shown in Figure 5 of the main text, but for photonic lasing. Just like in our plasmonic lasers, our photonic lasers show multi-mode lasing when the entire cavity is optically excited
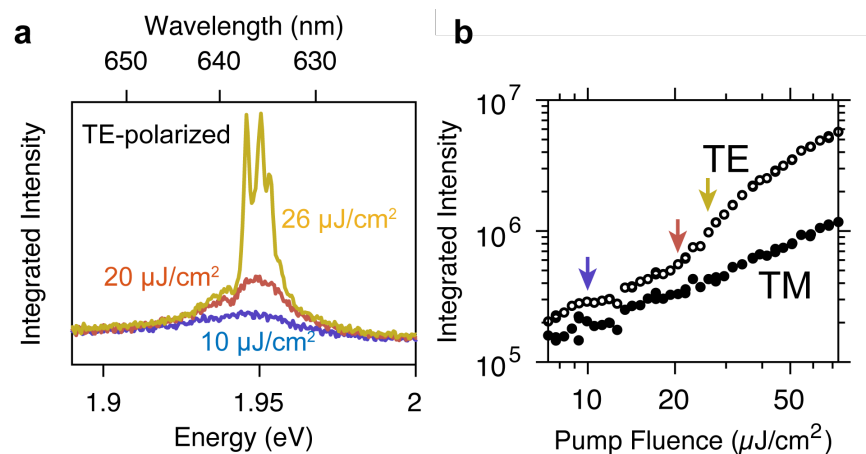

Figure S6. Lasing characteristics of a photonic device. a) Similar to Figure 1 in the main text, the emission spectrum shows purely spontaneous emission below the lasing threshold and consists of several sharp peaks above the threshold, which correspond to the different transverse and longitudinal modes of the cavity. b) If the NPL film is thick enough to support photonic TE modes, these become the dominant lasing mode as seen by the predominant TE polarization above the lasing threshold. For photonic lasing, we observe a more pronounced "kink" in the power-power plot with a clearly visible threshold. 


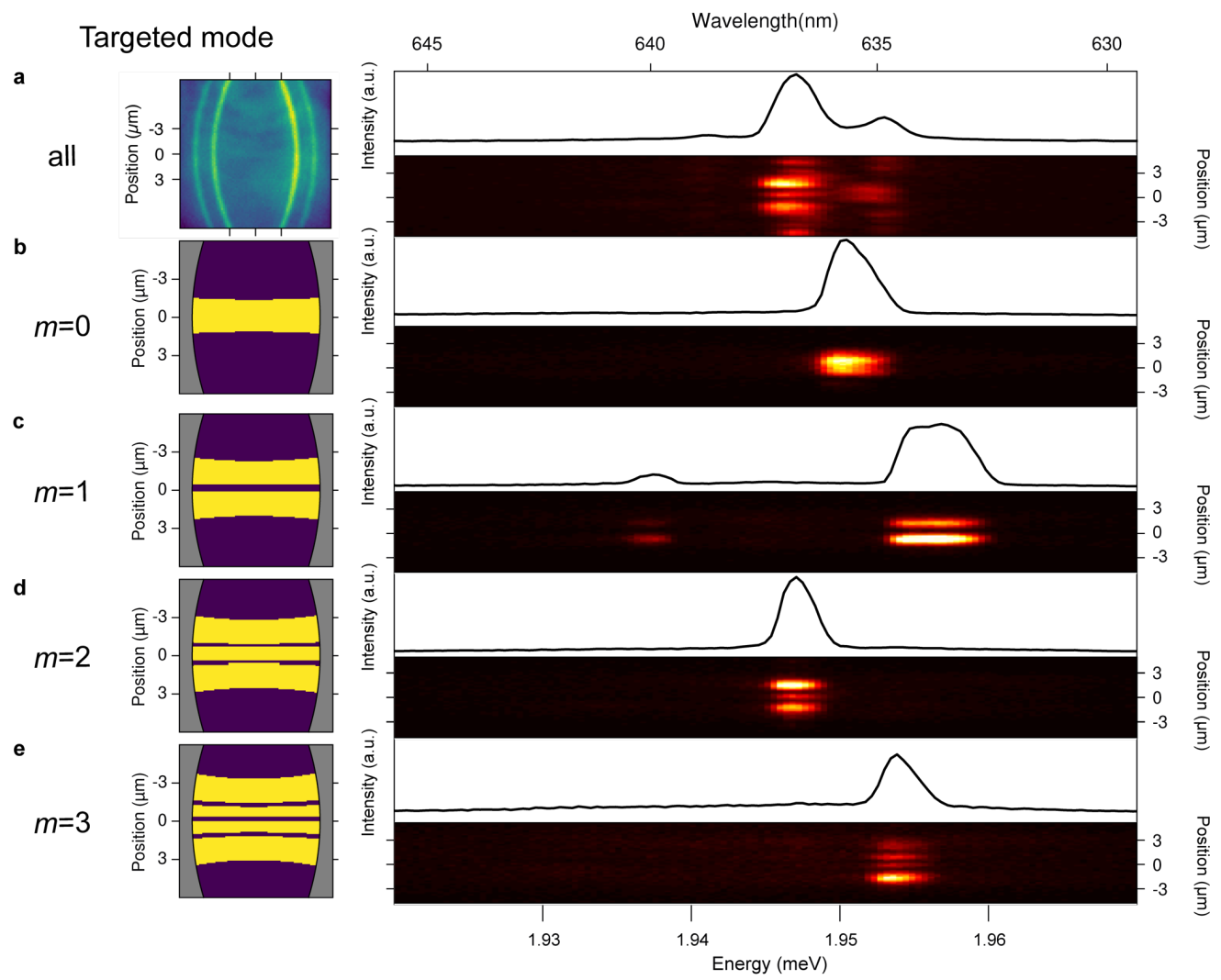

Figure S7. Mode-switching experiment for photonic lasing, analogous to the results shown for plasmonic lasing in Figure 5 of the main text. To support photonic modes, the gain-layer thickness was increased to 90$100 \mathrm{~nm}$. For each row, we again plot the pump pattern (left panel), spatially resolved spectrum (lower right panel), and spatially integrated spectrum (upper right panel) for targeting the first four transverse modes. (a) Spatially homogeneous illumination excites several transverse modes and yields a complicated spatial intensity distribution. (b) Selective excitation of the $m=0$ mode results in single-mode lasing. (c) For the $m=1$ pump profile, two longitudinal modes with the same transverse mode number are within the gain envelope of the nanoplatelets. (d) For the $m=2$ pump profile, again we observe a single active mode with energy in between that of the $m=1$ and the next $m=0$ mode, as predicted by theory. (e) Finally, the $m=3$ transverse mode here is slightly shifted in energy compared to the fundamental $m=0$ mode. The presence of three nodes in the spatial distribution of the outscattered photons confirms successful excitation of the $m=3$ transverse mode.

(Figure S9a). For the photonic-lasing experiment, we derived excitation patterns based on the mode index calculated for photonic TE modes (see Section S3 above). Despite similar spatial intensity distributions, the differing mode index has an impact on the lateral extent of the patterns. Excitation with a pattern optimized for the $m=0$ mode results in a single lasing peak at $1.950 \mathrm{eV}$ that displays no minimum along the transverse axis (Figure S9b). When selectively exciting the $m=1$ mode, two lasing peaks are visible at 1.938 and $1.957 \mathrm{eV}$, respectively. The single minimum in the spatial intensity distribution proves that these are indeed both $m=1$ modes with different longitudinal mode numbers (Figure S9c). For the $m=2$ pump profile, again we observe a single active mode with energy in between that of the $m=1$ and the next $m=0$ mode, as predicted by theory (Figure S9d). The presence of two minima along the transverse axis is in agreement with this assignment. Finally, the $m=3$ transverse mode is isolated but slightly shifted in energy compared to the fundamental $m=0$ mode (Figure S9e). This difference might come from a pump-dependent change in the 
refractive index of the nanoplatelets that shifts the lasing peaks and becomes more pronounced at increasing pump fluences. The presence of three nodes in the spatial distribution of the outscattered photons again confirms successful excitation of the $m=3$ transverse mode.

\section{Section S10.Supplementary References}

(S1) Kress, S. J. P.; Cui, J.; Rohner, P.; Kim, D. K.; Antolinez, F. V.; Zaininger, K.-A.; Jayanti, S. V.; Richner, P.; McPeak, K. M.; Poulikakos, D.; Norris, D. J. A Customizable Class of Colloidal-QuantumDot Spasers and Plasmonic Amplifiers. Sci. Adv. 2017, 3, e1700688.

(S2) Rossinelli, A. A.; Rojo, H.; Mule, A. S.; Aellen, M.; Cocina, A.; De Leo, E.; Schäublin, R.; Norris, D. J. Compositional Grading for Efficient and Narrowband Emission in CdSe-Based Core/Shell Nanoplatelets. Chem. Mater. 2019, 31, 9567-9578.

(S3) Aellen, M.; Rossinelli, A. A.; Keitel, R. C.; Brechbühler, R.; Antolinez, F. V.; Cui, J.; Norris, D. J. Reconsidering the Design of Planar Plasmonic Lasers: Gain, Gap Layers, and Mode Competition. arXiv e-prints 2021, arXiv:2101.05881.

(S4) Samuel, I. D.; Namdas, E. B.; Turnbull, G. A. How to Recognize Lasing. Nat. Photonics 2009, 3, 546549.

(S5) Verhagen, E. Subwavelength Light Confinement with Surface Plasmon Polaritons. Dissertation, Utrecht University, 2009. 